\section{Enfermería y la atención primaria de salud}

\author{
Dianne Sofia Gonzalez Escobar*
}

Hace 40 años la Organización Mundial de la Salud (OMS) definió la Atención Primaria de Salud ( APS) como la estrategia para alcanzar la meta Salud para Todos en el año 2000, buscando implementar cambios significativos en la prestación de servicios de salud y en el nivel de bienestar de la población, especialmente de la más afectada por las inequidades políticas, sociales y económicas, y con mayor afectación en los factores determinantes de la salud (1). La Declaración de Alma Ata definió la APS como:

"La asistencia sanitaria esencial basada en métodos y tecnologías prácticos, científicamente fundados y socialmente aceptables, puesta al alcance de todos los individuos y familias de la comunidad mediante su plena participación y a un costo que la comunidad y el país puedan soportar, en todas y cada una de las etapas de su desarrollo con un espíritu de autorresponsabilidad y autodeterminación. La atención primaria forma parte integrante tanto del sistema nacional de salud, del que constituye la función central y el núcleo principal, como del desarrollo social y económico global de la comunidad". (1)

A pesar de no alcanzar la meta planteada, el desarrollo de la atención primaria mostro evidencias de mejora en los indicadores de salud, eficiencia de los sistemas de salud y reducción en las desigualdades (2-4).

En septiembre de 2000 la Organización de las Naciones Unidas (ONU) aprueba la Declaración del Milenio, comprometiendo a los países con una nueva alianza mundial para reducir los niveles de extrema pobreza y estableciendo una serie de objetivos sujetos a plazo, conocidos como los Objetivos de Desarrollo del Milenio(ODS), cuyo plazo de cumplimiento era el año 2015. La identificación del poco progreso alcanzado a la mitad del periodo de cumplimiento, las dificultades presentadas en su desarrollo y la amplitud de la problemática mundial relacionada con la pobreza, el hambre y las enfermedades, llevan a la renovación del compromiso de la APS considerando que sus valores, principios y planteamientos orientan el desarrollo de los sistemas de salud con reformas en pro de la cobertura universal, de la prestación de servicios, de políticas públicas y liderazgo, que involucran servicios y factores determinantes de salud como la educación, la vivienda, los ingresos y el medio ambiente (5).

En el 2014, la Organización Panamericana de la Salud y la OMS plantean la Estrategia para acceso universal a la salud y la cobertura universal de salud, que reitera el derecho a la salud, la solidaridad y la equidad, y promueve el desarrollo de sistemas de salud basados en la APS. En la Estrategia se establece que "las poblaciones en situación vulnerable, en extremos de la vida, las mujeres, los niños y niñas, las minorías étnicas, las poblaciones indígenas y afrodescendientes, los migrantes, los pacientes con enfermedades crónicas o incapacitantes, entre otros, son los grupos más afectados por este problema" (6).

Posteriormente, en septiembre de 2015 se desarrolló la Cumbre del Desarrollo Sostenible en la que se aprobó la Agenda 2030, que contiene los 17 Objetivos de Desarrollo Sostenible (ODS), de aplicación universal que rigen los esfuerzos de los países para el logro de un mundo sostenible en 2030 (7). Estos objetivos son herederos de los ODM y buscan ampliar los resultados alcanzados con ellos, así como conseguir las metas no logradas. Estos nuevos objetivos tienen la particularidad de convocar a todos los países a implementar medidas que promuevan la prosperidad protegiendo simultáneamente el planeta. Plantean que las estrategias para acabar con la pobreza deben ir acompañadas de procesos que promuevan el crecimiento económico y la intervención de necesidades sociales fundamentales como la educación, la salud, la protección social y el empleo, a la vez que se protege el medio ambiente y se lucha contra el cambio climático.

Los recursos humanos en salud, han sido un elemento esencial a lo largo de la implementación de todas la políticas, estrategias y planes que se han definido para el mejoramiento de las condiciones de salud y de vida de la población, así como la preocupación por su adecuado desarrollo, disponibilidad y calificación para la atención de las necesidades de la población. Actualmente, se considera que los recursos humanos para

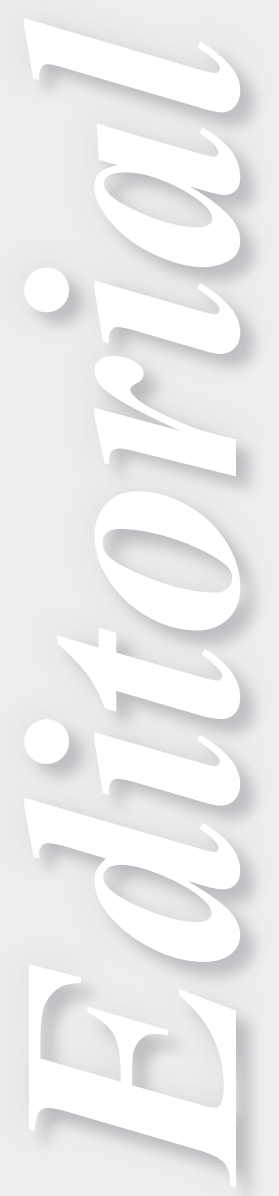

Autor de correspondencia

$\bowtie$ Enfermera. Magister en Enfermería. Magister en Educación de Adultos. Universidad Francisco de Paula Santander. E-mail: diannegonzales@ ufps.edu.co Orcid: (iD) https://orcid. org/0000-00028760-1743 Cúcuta, Colombia. 
1794-9831

E-ISSN 2322-7028

Vol. 16 No. 1

Ene - Abr 2019

Cúcuta, Colombia la salud son fundamentales para el logro de los ODS. La Asamblea Mundial de la Salud reconoció que las metas se lograrán si existe una inversión estratégica y sustantiva en los recursos humanos, considerando que los sistemas públicos de salud no están capacitando ni reclutando al número suficiente de trabajadores de salud, lo cual es indispensable para mejorar la capacidad resolutiva del primer nivel de atención (8).

Actualmente en América Latina se reconoce un déficit global de recursos humanos para la salud debido a la transición epidemiológica que se viene presentando a nivel mundial y en la región desde hace varias décadas, a la redistribución de la carga de la discapacidad y la mala distribución y migración de los profesionales de la salud (8). La OMS ha estimado que para el año 2035, el déficit mundial de trabajadores de la salud será de 12,9 millones, actualmente este déficit es de alrededor de 7,2 millones, de los cuales 4,3 millones corresponden a los médicos y a las enfermeras, afectando principalmente a los países en desarrollo (9).

Históricamente enfermería ha contribuido al desarrollo, implementación y funcionamiento de los sistemas de salud, desde Alma Ata ha sido enfermería quien ha liderado la implementación de programas de atención que responden a las prioridades definidas por las políticas de salud en nuestro país y en general, en los países de América Latina. Sin embargo, esta contribución más allá de su mención en documentos de diferentes organizaciones, no se ha reflejado en cambios en la estructuración de los servicios, la regulación del ejercicio profesional, los aspectos salariales y la participación del gremio en el decisorio político, entre otros.

En muchos países de la Región de las Américas, el ejercicio profesional de enfermería en los diferentes ámbitos de la atención en salud, entre ellos la APS, está subordinada al médico y tiene una limitada autonomía profesional; la atención del paciente en todos los niveles está en manos de técnicos y auxiliares de enfermería, y las enfermeras a pesar de ser las más calificadas por su formación están dedicadas a las actividades gerenciales, enfocando su rol, principalmente a la gestión administrativa del cuidado y no al cuidado directo del paciente (10). En Colombia, la revisión de la literatura realizada por De Arco y Suarez, evidencia que a pesar del rol que desempeñan los profesionales de enfermería, no se observa una diferenciación de perfiles y funciones dentro del equipo sanitario, lo que hace necesario la delimitación de funciones, la recuperación campos de acción, el fortalecimiento del liderazgo y la autonomía, y la humanización en la prestación de los servicios (11).

La situación de salud, las necesidades de la población y las problemáticas sociales, políticas, ambientales y económicas que demarcan las políticas de salud y los objetivos de desarrollo, impulsando la renovación de la atención primaria de salud para asegurar la cobertura y salud universal, llaman a enfermería a la reflexión y la acción como disciplina profesional y fuerza de trabajo mayoritaria en el sector de salud.

Retomando los planteamientos del Consejo Internacional de Enfermería( CIE ), "El ámbito de la práctica de enfermería no se limita a determinadas tareas, funciones o responsabilidades, sino que es una combinación de conocimientos, juicios y capacidades que permiten a la enfermera de llevar a cabo la prestación de cuidados directos y de evaluar sus resultados, de defender los pacientes y la salud, de supervisar y delegar en otros, de dirigir, gestionar, enseñar, realizar investigaciones y de desarrollar una política de salud para los sistemas de atención de salud. El ámbito de la práctica es dinámico y responde a las necesidades cambiantes de salud, .al desarrollo del conocimiento y a los avances tecnológicos" (12). Lo anterior, permite señalar que se hace indispensable la evaluación de los ámbitos de la práctica en el ejercicio del cuidado, para asegurar la pertinencia de éstos, no solo frente a las necesidades de salud sino a la naturaleza, competencia y razón social de la disciplina y profesión.

El cuidado representa la esencia de la práctica profesional, la cotidianidad del cuidado en APS al igual que en todos los ámbitos, implica no solo el conjunto de conocimientos, habilidades y actitudes propias, sino la apropiación y desarrollo de valores éticos, humanitarios y ciudadanos que permitan implementar y validar nuevas formas de brindar cuidado, centrado en la persona y promoviendo la integración, integralidad y continuidad de la atención en salud( 13). Por tanto, el trabajo de enfermería en APS, además de contribuir a la Salud Universal, representa un escenario para el desarrollo de procesos de mejoramiento en la formación, la práctica y la investigación en los diferentes contextos, así como el fortalecimiento del liderazgo y empoderamiento profesional como actores visibles en los espacios de decisorio político y toma de decisiones. 


\section{Referencias Bibliográficas}

1. Organización Mundial de la Salud (OMS). Conferencia Internacional sobre Atención Primaria de Salud, Alma-Ata, URSS [Internet]. 1978 [consultado 10 de Diciembre 2018] Disponible en: https:// www.paho.org/hq/dmdocuments/2012/Alma-Ata-1978Declaracion.pdf

2. Organización Panamericana de salud. Atención Primaria de Salud a 25 Años de la Declaración de Alma-Ata. Preguntas y respuestas. [Consultado el 4 de diciembre del 2018]. Disponible en http:// www.paho.org/spanish/dd/pin/alma preguntas.htm

3. Tirado Pedregosa G. De la Declaración de Alma-Ata 1978, al Informe sobre la Salud en el Mundo 2008. Enfermería Comunitaria [Internet] 2008 [Consultado 13 diciembre 2018] 4(2). Disponible en: http://www.index-f.com/comunitaria/v4n2/ec0342.php

4. Dedeu A, Lapena C, Marti T, Monguet JM, Picas JM. Reflexiones sobre la atención primaria. Documento de trabajo 172 [Internet]. 2011 [Consultado 10 de Diciembre 2018]. Disponible en: http://www. enfermeriacomunitaria.org/web/attachments/article/272/Reflexiones\%20sobre\%20APS.pdf

5. Organización Mundial de la Salud (OMS). Informe de la Salud en el mundo 2008, La atención primaria de salud, más necesaria que nunca [Internet]. 2008 [Consultado 10 de Diciembre 2018] Disponible en: https://www.who.int/whr/2008/whr08_en.pdf

6. Pan American Health Organization. Strategy for universal access to health and universal health coverage. [CD53.R14]. [Internet]. Washington, D.C.: OPS; 2014. [cited December 11, 2018]. Available from: https://www.paho.org/uhexchange/index.php/en/uhexchange-documents/technical-information/26strategy-for-universal-access-to-health-and-universal-health-coverage/file

7. Organización de Naciones Unidas (ONU). La Cubre de Desarrollo Sostenible 2015 [Internet]. 2015 [Consultado 13 Diciembre de 2018] Disponible en: https://www.who.int/mediacentre/events/meetings/2015/un-sustainable-development-summit/es/

8. Organización Mundial de la Salud, Organizacon Panamericana de la Salud. Estrategia de recursos humanos para el acceso universal a la salud y la cobertura universal de salud. 29. a Conferencia sanitaria panamericana. 69.a Sesión del Comité Regional de la OMS para las Américas. 2017.

9. Organización Mundial de la Salud. Working together for Health. Ginebra: OMS; 2006. Disponible en inglés: https://www.who.int/whr/2006/whr06_en.pdf

10. Organización Panamericana de la Salud. Ampliación del rol de las enfermeras y enfermeros en la atención primaria de salud. Washington, D.C.: OPS; 2018.

11. De Arco-Canoles OdelC, Suarez-Calle ZK. Rol de los profesionales de enfermería en el sistema de salud colombiano. Univ. Salud. 2018;20(2):171-182. DOI: http://dx.doi.org/10.22267/rus.182002.121

12. Consejo Internacional de Enfermeria. Ambito de la practica de Enfermera. Declaracion de posición. 2013. https://www.icn.ch/sites/default/files/inline-files/B07_Ambito_practica_enfermeria-Sp.pdf

13. Acioli S, Kebian LVA, Faria MGA, Ferraccioli P, Correa VAF. Care practices: the role of nurses in primary health care. Rev Enferm UERJ [Internet]. 2014 [Consultado 10 Diciembre de 2018]; 22(5):63742. Available from: http://www.e-publicacoes.uerj.br/index.php/ enfermagemuerj/article/view/12338 